\title{
Angiogenesehemmer verzögert Rezidiv beim Ovarialkarzinom
}

\begin{abstract}
Die positiven Ergebnisse der OCEANS-Studie haben 2012 zur Zulassung von Bevacizumab zur Rezidivtherapie des Ovarialkarzinoms geführt. Ein signifikant längeres Gesamtüberleben zeigt auch die aktuelle Auswertung nicht.
\end{abstract}

Ein ngeschlossen in die OCEANS-Studie waren 484 Patientinnen mit platinsensiblem Ovarialkarzinom, die nach der Erstlinientherapie mit Carboplatin/ Paclitaxel mindestens 6 Monate ohne Progress geblieben waren und Bevacizumab noch nicht in der Erstlinie erhalten hatten [Aghajanian $\mathrm{C}$ et al. J Clin Oncol. 2012;30(17):2039-45]. Sie bekamen Carboplatin/Gemcitabin über $6 \mathrm{Zy}$ klen und randomisiert entweder Bevacizumab oder Placebo; letztere wurden dann als Monotherapie bis zur Progression weitergegeben. Das progressionsfreie Überleben (PFS) war für diese $\mathrm{Pa}$ tientinnen unter Bevacizumab um vier Monate länger als in der Kontrollgruppe.

Die neuen Daten widmen sich den sekundären Endpunkten Sicherheit und Gesamtüberleben. Hier zeigten sich auch in der aktuellen Analyse der Studi- endaten keine signifikanten Unterschiede zwischen beiden Gruppen (33,6 vs. 32,9 Monate; Hazard Ratio 0,95).

Die detaillierte Aufschlüsselung in Sachen Sicherheit ergab, dass alle teilnehmenden Patientinnen mindestens 1 unerwünschtes Ereignis erlebten. Die Frauen in der Bevacizumab-Gruppe brachen die Studie aber wegen dieser Ereignisse häufiger ab als die Betroffenen der Kontrollgruppe (22,3 vs. 4,7\%). Wie von den Wissenschaftlern vorher postuliert, litten die Patientinnen der BevacizumabGruppe wesentlich häufiger unter Bluthochdruck (43,7 vs. 8,6\%), Proteinurie (21,5 vs. $4,3 \%)$ und Blutungen, die nicht das ZNS betrafen (68,0 vs. $32,6 \%)$. $1 \mathrm{~Pa}$ tientin in jeder Gruppe erlitt ein besonders schwerwiegendes Ereignis vom Grad 5: Unter Bevacizumab eine intrakranielle Blutung, unter Placebo einen
Myokard-Infarkt. Die jetzt vorliegenden Ergebnisse zeigen aber keine Hinweise auf neue Unverträglichkeitsmerkmale, die nicht schon 2012 veröffentlicht worden waren.

Auch wenn die aktuellen Daten in Bezug auf den sekundären Endpunkt Gesamtüberleben keinen signifikanten Unterschied zwischen den beiden Gruppen zeigen, zeichnet sich immerhin ein positiver Trend in der Bevacizumab-Gruppe hinsichtlich eines längeren progressionsfreien Überlebens ab.

Fazit: Obwohl die Patientinnen in der Bevacizumab-Gruppe insgesamt nicht signifikant länger überleben, tritt bei der Mehrzahl der Betroffenen ein Rezidiv erst später auf. Es wird also auch erst später eine weitere Therapie nötig. Heike Grosse

Aghajanian $C$ et al. Final overall survival and safety analyses of OCEANS, a phase 3 trial of chemotherapy with or without bevacizumab in patients with platin-sensitive recurrent cancer. Gynecol Oncol. 2015;139(1):10-6.

\section{Unerfüllter Kinderwunsch: subklinische Hypothyreose behandeln oder nicht?}

\section{Das Praxiskomitee der American Society for Reproductive Medicine (ASRM) hat eine Leitlinie zum Thema subklinische Hypothyreose bei Frauen mit uner- fülltem Kinderwunsch vorgelegt. Knallharte Empfehlungen enthält sie nicht.}

$E_{\text {re }}^{\text {in }}$ ine manifeste Hypothyreose kann die reproduktive Gesundheit von Frauen beeinträchtigen. Eine Schilddrüsenunterfunktion erhöht das Risiko für Infertilität, unter anderem aber auch für mehr Fehlgeburten oder Geburtskomplikationen. Es gibt Hinweise, dass sich auch eine subklinische Hypothyreose bzw. deren mangelnde Behandlung auf die Fruchtbarkeit auswirken können. Allerdings ist unklar, wann eine subklinische Hypothyreose vorliegt. Die Konzentration des freien Thyroxins sollte im Normbereich liegen, doch wie hoch darf der TSH-Wert sein? Setzt man die Obergrenze bei $5 \mu \mathrm{IU} / \mathrm{ml}$ an, hätten 4-8\% der Menschen im Reproduk- tionsalter eine subklinische Hypothyreose. Allerdings haben 95\% der Menschen ohne Schilddrüsenprobleme einen TSHWert unter 2,5 $\mu \mathrm{IU} / \mathrm{ml}$. Mit einer solchen Schwelle würden zusätzliche $12-14 \%$ der Bevölkerung in die Kategorie der subklinischen Hypothyreose fallen. Dennoch könnte der Wert für Frauen, die nicht schwanger werden, eine Schwelle zur Therapie darstellen. Die ASRM rät daher:

1. Der TSH-Spiegel infertiler Frauen ist zu messen. Oberhalb von $4 \mu \mathrm{IU} / \mathrm{ml}$ ist eine Levothyroxin-Therapie indiziert, um die Werte unter 2,5 $\mu \mathrm{IU} / \mathrm{ml}$ zu halten.

2. Liegen die TSH-Spiegel zwischen 2,5 und $4 \mu \mathrm{IU} / \mathrm{ml}$ sollte abgewartet und erst dann behandelt werden, sobald ein Wert von $4 \mu \mathrm{IU} / \mathrm{ml}$ überschritten ist; der Wert sollte mit Levothyroxin unter 2,5 $\mu \mathrm{IU} / \mathrm{ml}$ gesenkt werden.

3. Im ersten Schwangerschaftstrimester ist es ratsam, bereits bei Werten über 2,5 $\mu \mathrm{IU} / \mathrm{ml}$ zu behandeln.

4. Eine routinemäßige Schilddrüsenantikörper-Screening wird nicht empfohlen. Es kann aber erwogen werden, wenn die TSH-Werte wiederholt höher als 2,5 $\mathrm{IIU} / \mathrm{ml}$ liegen oder andere Risikofaktoren für Schilddrüsenerkrankungen vorliegen.

5. Bei Thyreoperoxidase-Antikörpern ist der TSH-Spiegel zu überprüfen. Ab 2,5 $\mu \mathrm{IU} / \mathrm{ml}$ ist eine Therapie zu erwägen.

Die Nachweislage der Empfehlungen 1, 3 und 5 (Grad B) ist ausreichend, bei 2 und 4 (Grad C) mangelhaft. Dr. Robert Bublak

Practice Committee of the American Society for Reproductive Medicine. Subclinical hypothyroidism in the infertile female population: a guideline. Fertil Steril 2015; online 31. Juli; doi: 10.1016/j.fertnstert.2015.05.028 